\title{
A new sight: topology-dependent mitophagy
}

\author{
Yanshuang Zhou • Qi Long • Xingguo Liu
}

Received: 2 March 2020 / Accepted: 30 May 2020 /Published online: 11 June 2020

(C) Springer Nature B.V. 2020

\begin{tabular}{|c|c|}
\hline \multicolumn{2}{|l|}{ Abbreviations } \\
\hline BCL2L13 & BCL2-like 13 \\
\hline BNIP3 & BCL2-interacting protein 3 \\
\hline BNIP3L/NIX & $\begin{array}{l}\text { BCL2-interacting protein } 3 \\
\text { like }\end{array}$ \\
\hline CALCOCO2/NDP52 & $\begin{array}{l}\text { Calcium-binding and } \\
\text { coiled-coil domain } 2\end{array}$ \\
\hline FUNDC1 & FUN14 domain-containing 1 \\
\hline GABARAP & $\begin{array}{l}\text { Gamma-aminobutyric acid } \\
\text { receptor-associated protein }\end{array}$ \\
\hline LIR & LC3-interacting region \\
\hline MAP 1LC3/LC3 & $\begin{array}{l}\text { Microtubule-associated } \\
\text { protein } 1 \text { light chain } 3\end{array}$ \\
\hline MFN1 & mitofusin 1 \\
\hline MFN2 & Mitofusin 2 \\
\hline mPTP & $\begin{array}{l}\text { Mitochondrial permeability } \\
\text { transition pore }\end{array}$ \\
\hline
\end{tabular}

Yanshuang Zhou and Qi Long contributed equally to this work.

Y. Zhou · Q. Long · X. Liu

Guangzhou Regenerative Medicine and Health Guangdong Laboratory, CAS Key Laboratory of Regenerative Biology, Joint School of Life Sciences, Hefei Institute of Stem Cell and Regenerative Medicine, Guangzhou Institutes of Biomedicine and Health, Chinese Academy of Sciences; Guangzhou Medical University, Guangzhou 510530, China

Y. Zhou $\cdot$ Q. Long $\cdot$ X. Liu $(\bowtie)$

Guangdong Provincial Key Laboratory of Stem Cell and Regenerative Medicine, Institute for Stem Cell and Regeneration, Guangzhou Institutes of Biomedicine and Health,University of Chinese Academy of Sciences, Chinese Academy of Sciences, Guangzhou 510530, China

e-mail: liu_xingguo@gibh.ac.cn

$\begin{array}{ll}\text { NBR1 } & \begin{array}{l}\text { NBR1 autophagy cargo } \\ \text { receptor }\end{array} \\ \text { OPTN } & \text { Optineurin } \\ \text { PHB2 } & \text { Prohibitin 2 } \\ \text { PINK1 } & \text { PTEN-induced putative } \\ & \text { kinase 1 } \\ \text { parkin RBR E3 ubiquitin } \\ \text { PRKN/PARKIN } & \text { protein ligase } \\ \text { P62/SQSTM1 } & \text { Sequestosome 1 } \\ \text { ROS } & \text { Reactive oxygen species } \\ \text { TBK1 } & \text { Tank-binding kinase 1 } \\ \Delta \Psi_{\mathrm{m}} & \text { Mitochondrial membrane } \\ & \text { potential }\end{array}$

Mitochondria play an essential role as an energy ATP producer. In addition, mitochondria participate in various biological processes, such as apoptosis, reactive oxygen species (ROS) production, and $\mathrm{Ca}^{2+}$ signal. The elimination of damaged or unwanted mitochondria is essential to maintain the mitochondrial network function and cellular homeostasis. Currently, many pieces of evidence demonstrate that mitophagy, a selective autophagy for mitochondria, is critical for the developmental process, cell fate determination, and stress response (Song et al. 2016; Green and Levine 2014; Esteban-Martinez et al. 2017; Wu et al. 2016). The defects in mitophagy result in accumulation of damaged mitochondria, which is tightly associated with various diseases, such as heart failure (Shires and Gustafsson 2015; Zhou et al. 2020a), neurodegenerative disease (Reddy and Oliver 2019; Oliver and Reddy 2019), and aging-related diseases (Ding and Yin 2012). 
Mitophagy plays a vital role in the maintenance of mitochondrial quality and quantity. Mitophagy is a multi-step process: (1) recognition of damaged or unwanted mitochondria; (2) engulfment by the phagophore, resulting in the formation of mitophagosomes; (3) fusion with lysosomes for recycling the contents. It is important to study the mechanisms and principles in selective mitophagy, which can expand our knowledge to understand mitochondrial function as well as their roles in diseases. Several mechanisms have been identified as responsible for specific recognition and selective elimination of damaged or unwanted mitochondria, such as the PINK1 (PTEN induced kinase 1)-PRKN/PARKIN (parkin RBR E3 ubiquitin protein ligase) pathway and receptormediated pathway.

The PINK1-PRKN can recognize depolarized mitochondria and add ubiquitin chains on mitochondrial membrane proteins to amplify and recruit autophagy receptors (Narendra et al. 2010; Jin et al. 2010; Lazarou et al. 2012; Matsuda et al. 2010). These receptors serve as a bridge between cargos and MAP1LC3/ LC3 (microtubule-associated protein 1 light chain 3)
(Svenning and Johansen 2013; Stolz et al. 2014). Lazarou $\mathrm{M}$ et al. found that OPTN (Optineurin) and CALCOCO2/NDP52 (calcium-binding and coiled-coil domain 2 ) are the primary autophagy receptors, while P62/SQSTM1 (sequestosome 1) and NBR1 (NBR1 autophagy cargo receptor) are dispensable in PINK1PRKN-mediated mitophagy (Lazarou et al. 2015). What's more, PHB2 (prohibitin 2), an inner mitochondrial membrane protein, is required for PINK1-PRKNmediated mitophagy. PHB2 recruits LC3 through its LC3-interacting region (LIR) motif and promotes mitophagy after outer mitochondrial membrane rupture (Wei et al. 2017).

Besides the PINK1-PRKN pathway, mitophagy receptors such as BNIP3 (BCL2-interacting protein 3), BNIP3L/NIX (BCL2-interacting protein 3 like), FUNDC1 (FUN14 domain-containing 1), and BCL2L13 (BCL2-like 13), can mark damaged or unwanted mitochondria under mitophagic stimulus. These receptors that contain LIR motif can directly interact with LC3 or GABARAP (gammaaminobutyric acid receptor-associated protein) to induce mitophagy (Hamacher-Brady and Brady
Fig. 1 Selective elimination of mitochondria by PINK1-PRKN pathway and receptor-mediated pathway

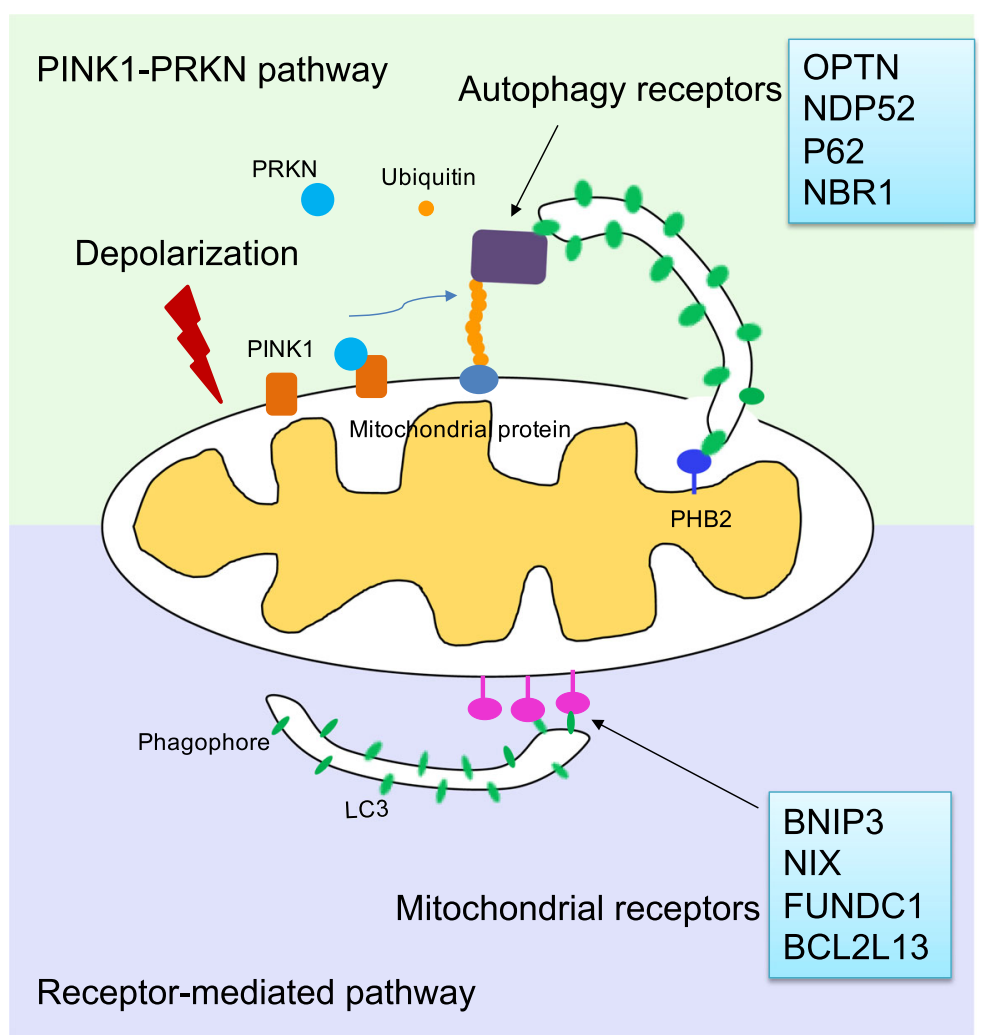


2016; Liu et al. 2014). Taken together, the selection of mitophagy is dependent on the accumulation of specific protein on damaged or unwanted mitochondria in the molecular level (Fig. 1).

Mitophagy and mitochondrial morphology, regulated by fusion and fission, are closely linked. Mitochondrial fission generates smaller mitochondria in size, which is easily enveloped by autophagosomes, while mitochondrial fusion generates elongated mitochondria (Gomes et al. 2011; Rambold et al. 2011; Okamoto et al. 2009; Twig et al. 2008). Given that the limited size of autophagosomes ranges from 0.5 to $1.5 \mu \mathrm{m}$ (Pfeifer 1978; Schworer et al. 1981; Mizushima and Klionsky 2007), we take it granted that mitochondrial division precedes engulfment during mitophagy. However, Yamashita et al. showed that mitochondrial division could occur concomitantly with autophagosome formation. Autophagosome formation factors are essential for mitochondrial division to enable enwrapping of the maximum size of mitochondria to fit into autophagosome (Yamashita et al. 2016). Taken together, these findings demonstrate the importance of mitochondrial topology in mitophagosome engulfment. Recently, our lab showed a topology-dependent, bifurcated mitochondrial quality control model (Zhou et al. 2020b), which enriches the contents of selective mitophagy in organellar morphology-dependent manner (Fig. 2).
We found that mitochondria undergo different topological transformations during starvation, either swelling or forming donut shapes. Swollen mitochondria are associated with mitochondrial membrane potential $\left(\Delta \Psi_{\mathrm{m}}\right)$ dissipation and PRKN recruitment, resulting in the selective elimination by autophagy, while the donut topology maintains $\Delta \Psi_{\mathrm{m}}$ and helps mitochondria resist autophagy. Importantly, donut mitochondria can undergo fission and fusion cycles, which could recover to tubular one. Mechanistically, donut topology resists autophagy even after depolarization, wherein the recruitment of autophagosome receptors CALCOCO2 and OPTN after PRKN recruitment and ubiquitination is prevented.

It would be interesting to investigate how donut mitochondria prevent mitophagy after outer membrane protein ubiquitination. One important issue may be the integrity of mitochondrial structure which guarantees the function of mitochondria after topology transformation. The crista structure is one of the fundamental structures for the oxidative phosphorylation system. We showed that the crista structure maintains well in donut mitochondria, while it is not so abundant in swollen ones, indicating the functional integrity of the donut structure. A mitophagy receptor PHB2 localized in crista may be exposed in swollen mitochondria as the structure rupture and mediate mitophagy in the PINKPARKN pathway. Furthermore, as the externalization of
Fig. 2 Mitochondrial topology and mitophagy

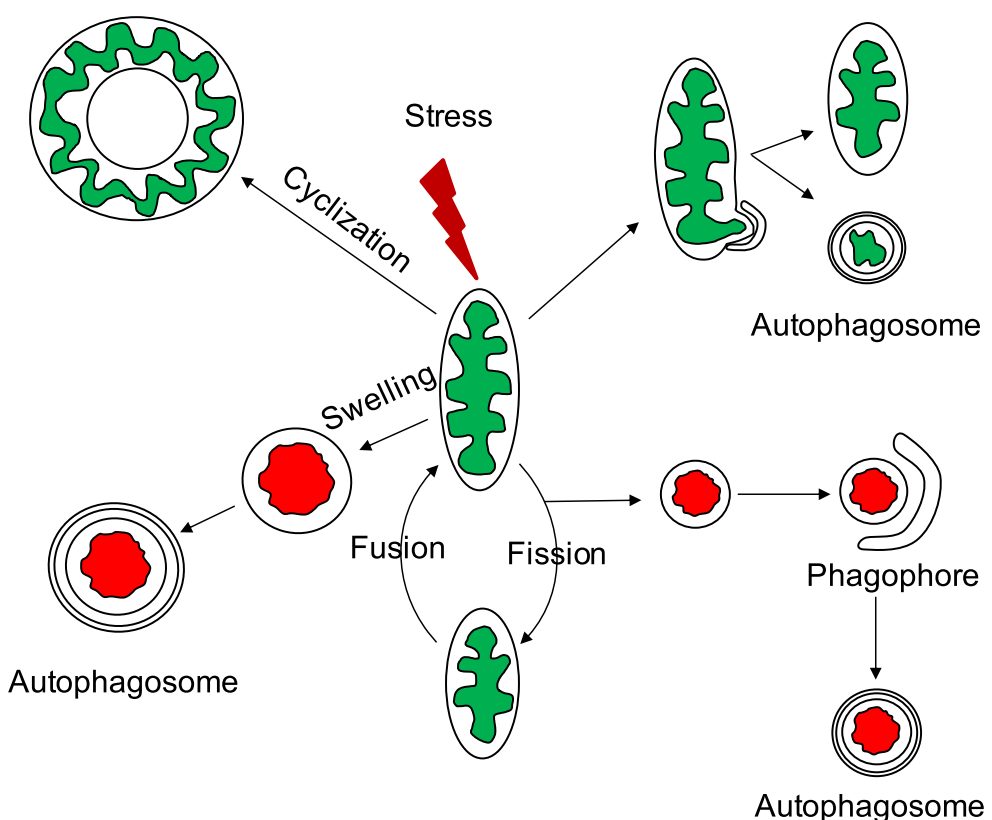

Autophagosome 
cardiolipin to the outer mitochondrial membrane is a mitophagy signal, it would be interesting to observe the distribution of cardiolipin in donut and swollen mitochondria. LC3 contains cardiolipin-binding sites, which is important for the engulfment of mitochondria by the autophagic system (Chu et al. 2013). We hypothesize that cardiolipin is not transported to the donut mitochondrial surface; thus, it could not be enveloped by LC3 even though ubiquitination.

Donut mitochondria have been described long time ago (Lauber 1982; Funk et al. 1999), and the roles of the outer mitochondrial membrane localized GTPases (mitofusin 1/2) were initially described in yeast (Mozdy and Shaw 2003; Messerschmitt et al. 2003; Hermann et al. 1998). Similarly, we also showed that mitochondrial fusion is indispensable for donuts, for which no donuts are observed in mitofusin 1/2 double-knockout ( $\mathrm{mfn} 1 / 2$ KO) MEF cells. Donut-shaped mitochondria, not swollen mitochondria, are reversible to linear one. We also discussed the formation of donuts, which is triggered by the opening of mitochondrial permeability transition pore (mPTP) or $\mathrm{K}^{+}$channels, and as a result of osmotic pressure changes. We showed the extent of channel opening is very important in producing both donuts and swelling. For example, valinomycin, a $\mathrm{K}^{+}$ionophore, induced mitochondrial swelling, and donut formation at high (5-500 $\mathrm{nM})$ and low $(0.25-0.5 \mathrm{nM})$ concentration, respectively. What's more, donut mitochondria are able to tolerate the swelling volume of mitochondria than tubular mitochondria (Liu and Hajnoczky 2011). The Gibbs energy model of donut mitochondria we developed previously figures out that the donut is a lower-energy state compared with tubular one under stress (Long et al. 2015). In apoptosis, tubular mitochondria turn to swollen mitochondria after mitochondrial fission and swelling (Frank et al. 2001). Hence, to what extent the opening of channels plays a role in producing both types of mitochondria and to what extent both types of mitochondrial morphology are linked to the activation of apoptosis would be very interesting.

Donut mitochondria are observed in many physiological and pathological conditions, such as hypoxiareoxygenation (Liu and Hajnoczky 2011), neuron degeneration (Hara et al. 2014), aging (Lauber 1982; Hara et al. 2014), and osteosarcoma (Jackson et al. 2018). The formation of donut has been suggested to be a hallmark of stress (Picard and McEwen 2014). Ahmad T et al. provided computational correlations of mitochondrial shape change with the level of mitochondrial ROS generated by cells. Mitochondria transform to donut under mild stress and to blob form during high oxidative stress (Ahmad et al. 2013). This is consistent with our result that the transition from tubular to donut is reversible. The appearance of donut mitochondria indicates that cells are in stress and could recover if the stress withdraws. On the other hand, with prolonged time of depolarization, donut mitochondria finally turn into swollen mitochondria.

The selective elimination of mitochondria in the molecular and organellar level is crosslinked (Yoo and Jung 2018). For example, PINK1-PRKN could separate damaged mitochondria within a small size from normal ones by inhibiting mitochondrial fusion, for which PRKN can ubiquitinate MFN1/2 (mitofusin 1/2) and activate ubiquitin-proteasome system (Gegg et al. 2010). BCL2L13 not only serves as a receptor of mitophagy but also takes part in mitochondrial fission (Murakawa et al. 2015). Similarly, FUNDC1 not only mediates mitophagy but also involves in mitochondrial fission (Liu et al. 2012; Wu et al. 2016; Chen et al. 2016). In addition, modification of many proteins involved in mitophagy by phosphatase and phosphokinase precisely controls the process of mitophagy. For example, Tank-binding kinase 1 (TBK1) phosphorylates OPTN at S473, which enhances its binding to ubiquitin chains and promotes selective autophagy of mitochondria (Richter et al. 2016).

The mechanism of mitophagy is regulated by a multilevel network that many mitochondrial and cellular machineries are involved in. We just briefly propose a new sight in mitochondrial topology-dependent model of mitophagy. The new model we constructed provides a new strategy in selective removing mitochondria in a topology- dependent manner. It indicates that the topology of mitochondria not only represents the state of mitochondria but also serves as a role in selective mitophagy. It needs further study in the interaction between mitochondrial topology and mitophagy, as well as its roles in contributing to the pathology of chronic diseases.

Acknowledgments We are grateful to all members in Professor Xingguo Liu's laboratory for useful discussions.

Funding information This study was funded by the National Key Research and Development Program of China (2018YFA0107100), the Strategic Priority Research Program of the Chinese Academy of Sciences (XDA16030505), the National Key Research and Development Program of China (2017YFA0106300, 2017YFA0102900, 2017YFC1001602, 2019YFA09004500, 2016YFA0100300), the National Natural Science Foundation projects of China (U1601227, 
31631163001, 31701281, 31701106, 31801168, 31900614, 31970709, 81901275), the Key Research Program of Frontier Sciences, CAS (QYZDB-SSW-SMC001), CAS STS Program KFJ-STS-QYZD-125, Guangzhou Health Care and Cooperative Innovation Major Project (201704020218), Guangdong Province Science and Technology Program (2017B020230005, 2017A020215056, 2017B030314056, 2018A030313825, 2018GZR110103002, 2020A1515011200, 2020A1515010919, 2020A1515011410), Guangzhou Science and Technology Program (201707010178, 201807010067, 202002030277), Grant from Yangtse River Scholar Bonus Schemes (to X. L.)

\section{Compliance with ethical standards}

Conflict of interest The authors declare that they have no competing interests.

\section{References}

Ahmad T, Aggarwal K, Pattnaik B, Mukherjee S, Sethi T, Tiwari $\mathrm{BK}$ et al. Computational classification of mitochondrial shapes reflects stress and redox state. Cell death \& disease. 2013;4:e461. https://doi.org/10.1038/cddis.2012.213.

Chen M, Chen Z, Wang Y, Tan Z, Zhu C, Li Y, et al. Mitophagy receptor FUNDC1 regulates mitochondrial dynamics and mitophagy. Autophagy. 2016;12(4):689-702. https://doi. org/10.1080/15548627.2016.1151580.

Chu CT, Ji J, Dagda RK, Jiang JF, Tyurina YY, Kapralov AA, et al. Cardiolipin externalization to the outer mitochondrial membrane acts as an elimination signal for mitophagy in neuronal cells. Nat Cell Biol. 2013;15(10):1197-205. https://doi.org/10.1038/ncb2837.

Ding WX, Yin XM. Mitophagy: mechanisms, pathophysiological roles, and analysis. Biol Chem. 2012;393(7):547-64. https://doi.org/10.1515/hsz-2012-0119.

Esteban-Martinez L, Sierra-Filardi E, Boya P. Mitophagy, metabolism, and cell fate. Mol Cell Oncol. 2017;4(5):e1353854. https://doi.org/10.1080/23723556.2017.1353854.

Frank S, Gaume B, Bergmann-Leitner ES, Leitner WW, Robert EG, Catez F, et al. The role of dynamin-related protein 1, a mediator of mitochondrial fission, in apoptosis. Dev Cell. 2001;1(4):515-25. https://doi.org/10.1016/s1534-5807(01 00055-7.

Funk RH, Nagel F, Wonka F, Krinke HE, Golfert F, Hofer A. Effects of heat shock on the functional morphology of cell organelles observed by video-enhanced microscopy. Anat Rec. 1999;255(4):458-64. https://doi.org/10.1002 /(sici)1097-0185(19990801)255:4<458::aid-ar11>3.0.co;2u.

Gegg ME, Cooper JM, Chau KY, Rojo M, Schapira AH, Taanman JW. Mitofusin 1 and mitofusin 2 are ubiquitinated in a PINK1/parkin-dependent manner upon induction of mitophagy. Hum Mol Genet. 2010;19(24):4861-70. https://doi.org/10.1093/hmg/ddq419.

Gomes LC, Di Benedetto G, Scorrano L. During autophagy mitochondria elongate, are spared from degradation and sustain cell viability. Nat Cell Biol. 2011;13(5):589-98. https://doi. org/10.1038/ncb2220.

Green DR, Levine B. To be or not to be? How selective autophagy and cell death govern cell fate. Cell. 2014;157(1):65-75. https://doi.org/10.1016/j.cell.2014.02.049.

Hamacher-Brady A, Brady NR. Mitophagy programs: mechanisms and physiological implications of mitochondrial targeting by autophagy. Cell Mol Life Sci. 2016;73(4):77595. https://doi.org/10.1007/s00018-015-2087-8.

Hara Y, Yuk F, Puri R, Janssen WG, Rapp PR, Morrison JH. Presynaptic mitochondrial morphology in monkey prefrontal cortex correlates with working memory and is improved with estrogen treatment. Proc Natl Acad Sci U S A 2014;111(1): 486-491. doi:https://doi.org/10.1073/pnas.1311310110.

Hermann GJ, Thatcher JW, Mills JP, Hales KG, Fuller MT, Nunnari J, et al. Mitochondrial fusion in yeast requires the transmembrane GTPase Fzolp. J Cell Biol. 1998;143(2): 359-73. https://doi.org/10.1083/jcb.143.2.359.

Jackson M, Serada N, Sheehan M, Srinivasan S, Mason N, Guha $\mathrm{M}$, et al. Mitochondrial genome and functional defects in osteosarcoma are associated with their aggressive phenotype. PloS One. 2018;13(12):e0209489-e. https://doi.org/10.1371 journal.pone.0209489.

Jin SM, Lazarou M, Wang C, Kane LA, Narendra DP, Youle RJ. Mitochondrial membrane potential regulates PINK1 import and proteolytic destabilization by PARL. J Cell Biol. 2010;191(5):933-42. https://doi.org/10.1083 jjcb.201008084.

Lauber JK. Retinal pigment epithelium: ring mitochondria and lesions induced by continuous light. Curr Eye Res. 1982;2(12):855-62. https://doi.org/10.3109 /02713688209020022.

Lazarou M, Jin SM, Kane LA, Youle RJ. Role of PINK1 binding to the TOM complex and alternate intracellular membranes in recruitment and activation of the E3 ligase Parkin. Dev Cell. 2012;22(2):320-33. https://doi.org/10.1016/j. devcel.2011.12.014.

Lazarou M, Sliter DA, Kane LA, Sarraf SA, Wang C, Burman JL, et al. The ubiquitin kinase PINK1 recruits autophagy receptors to induce mitophagy. Nature. 2015;524(7565):309-14. https://doi.org/10.1038/nature14893.

Liu X, Hajnoczky G. Altered fusion dynamics underlie unique morphological changes in mitochondria during hypoxiareoxygenation stress. Cell Death Differ. 2011;18(10):156172. https://doi.org/10.1038/cdd.2011.13.

Liu L, Feng D, Chen G, Chen M, Zheng Q, Song P, et al. Mitochondrial outer-membrane protein FUNDC1 mediates hypoxia-induced mitophagy in mammalian cells. Nat Cell Biol. 2012;14(2):177-85. https://doi.org/10.1038/ncb2422.

Liu L, Sakakibara K, Chen Q, Okamoto K. Receptor-mediated mitophagy in yeast and mammalian systems. Cell Res. 2014;24(7):787-95. https://doi.org/10.1038/cr.2014.75.

Long Q, Zhao D, Fan W, Yang L, Zhou Y, Qi J, et al. Modeling of mitochondrial donut formation. Biophys J. 2015;109(5): 892-9. https://doi.org/10.1016/j.bpj.2015.07.039.

Matsuda N, Sato S, Shiba K, Okatsu K, Saisho K, Gautier CA, et al. PINK1 stabilized by mitochondrial depolarization recruits Parkin to damaged mitochondria and activates latent Parkin for mitophagy. J Cell Biol. 2010;189(2):211-21. https://doi.org/10.1083/jcb.200910140. 
Messerschmitt M, Jakobs S, Vogel F, Fritz S, Dimmer KS, Neupert $\mathrm{W}$, et al. The inner membrane protein Mdm33 controls mitochondrial morphology in yeast. J Cell Biol. 2003;160(4):553-64. https://doi.org/10.1083 /jcb.200211113.

Mizushima N, Klionsky DJ. Protein turnover via autophagy: implications for metabolism. Annual review of nutrition. 2007;27:19-40. https://doi.org/10.1146/annurev. nutr.27.061406.093749.

Mozdy AD, Shaw JM. A fuzzy mitochondrial fusion apparatus comes into focus. Nat Rev Mol Cell Biol. 2003;4(6):468-78. https://doi.org/10.1038/nrm1125.

Murakawa T, Yamaguchi O, Hashimoto A, Hikoso S, Takeda T, Oka T, et al. Bcl-2-like protein 13 is a mammalian Atg32 homologue that mediates mitophagy and mitochondrial fragmentation. Nat Commun. 2015;6:7527. https://doi. org/10.1038/ncomms8527.

Narendra DP, Jin SM, Tanaka A, Suen DF, Gautier CA, Shen J, et al. PINK1 is selectively stabilized on impaired mitochondria to activate Parkin. PLoS Biol. 2010;8(1):e1000298. https://doi.org/10.1371/journal.pbio.1000298.

Okamoto K, Kondo-Okamoto N, Ohsumi Y. Mitochondriaanchored receptor Atg32 mediates degradation of mitochondria via selective autophagy. Dev Cell. 2009;17(1):87-97. https://doi.org/10.1016/j.devcel.2009.06.013.

Oliver DMA, Reddy PH. Molecular Basis of Alzheimer's Disease: Focus on Mitochondria. J Alzheimer's Dis. 2019;72(s1): S95-s116. https://doi.org/10.3233/jad-190048.

Pfeifer U. Inhibition by insulin of the formation of autophagic vacuoles in rat liver. A morphometric approach to the kinetics of intracellular degradation by autophagy. J Cell Biol. 1978;78(1):152-67. https://doi.org/10.1083/jcb.78.1.152.

Picard M, McEwen BS. Mitochondria impact brain function and cognition. Proc Natl Acad Sci U S A 2014;111(1):7-8. doi: https://doi.org/10.1073/pnas.1321881111.

Rambold AS, Kostelecky B, Elia N, Lippincott-Schwartz J. Tubular network formation protects mitochondria from autophagosomal degradation during nutrient starvation. Proc Natl Acad Sci U S A. 2011;108(25):10190-5. https://doi.org/10.1073/pnas.1107402108.

Reddy PH, Oliver DM. Amyloid beta and phosphorylated Tauinduced defective autophagy and mitophagy in Alzheimer's disease. Cells. 2019;8(5). https://doi.org/10.3390 /cells 8050488 .

Richter B, Sliter DA, Herhaus L, Stolz A, Wang C, Beli P, et al. Phosphorylation of OPTN by TBK1 enhances its binding to $\mathrm{Ub}$ chains and promotes selective autophagy of damaged mitochondria. Proc Natl Acad Sci U S A. 2016;113(15): 4039-44. https://doi.org/10.1073/pnas.1523926113.

Schworer CM, Shiffer KA, Mortimore GE. Quantitative relationship between autophagy and proteolysis during graded amino acid deprivation in perfused rat liver. The Journal of biological chemistry. 1981;256(14):7652-8.

Shires SE, Gustafsson AB. Mitophagy and heart failure. J Mol Med (Berlin, Germany). 2015;93(3):253-62. https://doi. org/10.1007/s00109-015-1254-6.

Song WH, Yi YJ, Sutovsky M, Meyers S, Sutovsky P. Autophagy and ubiquitin-proteasome system contribute to sperm mitophagy after mammalian fertilization. Proc Natl Acad Sci U S A. 2016;113(36):E5261-70. https://doi.org/10.1073 /pnas.1605844113.

Stolz A, Ernst A, Dikic I. Cargo recognition and trafficking in selective autophagy. Nat Cell Biol. 2014;16(6):495-501. https://doi.org/10.1038/ncb2979.

Svenning S, Johansen T. Selective autophagy. Essays Biochem. 2013;55:79-92. https://doi.org/10.1042/bse0550079.

Twig G, Elorza A, Molina AJ, Mohamed H, Wikstrom JD, Walzer $\mathrm{G}$, et al. Fission and selective fusion govern mitochondrial segregation and elimination by autophagy. EMBO J. 2008;27(2):433-46. https://doi.org/10.1038/sj. emboj.7601963.

Wei Y, Chiang WC, Sumpter R Jr, Mishra P, Levine B. Prohibitin 2 is an inner mitochondrial membrane mitophagy receptor. Cell. 2017;168(1-2):224-38.e10. https://doi.org/10.1016/j. cell.2016.11.042.

Wu W, Li W, Chen H, Jiang L, Zhu R, Feng D. FUNDC1 is a novel mitochondrial-associated-membrane (MAM) protein required for hypoxia-induced mitochondrial fission and mitophagy. Autophagy. 2016;12(9):1675-6. https://doi. org/10.1080/15548627.2016.1193656.

Yamashita SI, Jin X, Furukawa K, Hamasaki M, Nezu A, Otera H, et al. Mitochondrial division occurs concurrently with autophagosome formation but independently of Drp1 during mitophagy. J Cell Biol. 2016;215(5):649-65. https://doi. org/10.1083/jcb.201605093.

Yoo SM, Jung YK. A Molecular approach to mitophagy and mitochondrial dynamics. Mol Cells. 2018;41(1):18-26. https://doi.org/10.14348/molcells.2018.2277.

Zhou H, He L, Xu G, Chen L. Mitophagy in cardiovascular disease. Clin Chim Acta. 2020a. https://doi.org/10.1016/j. cca.2020.04.033.

Zhou Y, Long Q, Wu H, Li W, Qi J, Wu Y, et al. Topologydependent, bifurcated mitochondrial quality control under starvation. Autophagy. 2020b;16(3):562-74. https://doi. org/10.1080/15548627.2019.1634944.

Publisher's note Springer Nature remains neutral with regard to jurisdictional claims in published maps and institutional affiliations. 